\title{
Stability of Jurisdiction Structures under the Equal Share and Median Rules
}

\author{
Anna Bogomolnaia* $\quad$ Michel Le Breton ${ }^{\dagger} \quad$ Alexei Savvateev ${ }^{\ddagger}$ \\ Shlomo Weber ${ }^{\S}$
}

April 2005

\begin{abstract}
In this paper we consider a model with multiple jurisdictions where each formed jurisdiction selects a public project from the given uni-dimensional set, equally shares its cost among its members and places the project at the location of its median resident. We examine a cooperative concept of core stability and a non-cooperative notion of Nash stability of jurisdiction structures. We first consider hedonic games, where each jurisdiction chooses the mean of the extreme points of its median set, and show that both stability notions may lead to an empty set of stable jurisdiction structures. We demonstrate that in a quasi-hedonic set-up, where jurisdictions are allowed to pick any selection from the median set, there is a Nash stable partition, while a core stable partition may still fail to exist. We also consider the equidistant societies and show the existence of both types of stable partitions in this case. Finally, we examine stratification properties of stable partitions.
\end{abstract}

Keywords: Equal share rule, Median rule, Jurisdictions, Core and Nash stable partitions.

JEL Classification Numbers: C71, C72, D63, H41.

*Rice University, Houston, USA.

${ }^{\dagger}$ Université de Toulouse I, GREMAQ and IDEI, Toulouse, France.

${ }^{\ddagger}$ Central Economics and Mathematics Institute, Moscow; Institute for Theoretical and Experimental Physics, Moscow; New Economic School, Moscow; CORE, Catholic University of Louvain-la-Neuve, Belgium. Financial support through grants R98-0631 from the Economic Education and Research Consortium, \# NSh-1939.2003.6 School Support, Russian Foundation for Basic Research No. 04-02-17227, and the Russian Science Support Foundation is gratefully acknowledged.

${ }^{\S}$ SMU, Dallas, USA; CORE, Catholic University of Louvain, Belgium; and CEPR. 


\section{Introduction}

We consider a model with a finite number of agents who form jurisdictions that partition the society into pairwise disjoint groups. Each jurisdiction selects a public project (Mas-Colell (1980)) from the given uni-dimensional set represented by an interval, and then shares the project cost among its members. The projects in our set-up are "horizontally differentiated", where the agents display distinct preferences over the project space. For simplicity, we choose a paradigm of geographical location of public projects (schools, hospitals, libraries), which serves as a parameter of horizontal differentiation.

Most of environments where agents can form groups to conduct some type of economic activity are characterized by the basic conflict between increasing returns to scale and heterogeneity of agents' characteristics and tastes. The increasing returns to scale tend to support the creation of large groups, even the grand coalition, whereas the group heterogeneity may tip the scale in favor of smaller groups. In general, the benefits of size are neither negligible nor unlimited and we may observe the emergence of group structures which consist of groups smaller than the grand coalition but larger than singletons. The group formation problem of the type described here contains three major components (see, e.g., Le Breton and Weber (2004)):

- composition of formed jurisdictions;

- project choices within each jurisdiction;

- the mechanism of sharing the project costs among agents within the same jurisdiction.

The analysis of stability of group formation is centered on the joint examination of these three components that guarantee the stability of the jurisdiction structure under various forms of "secession" and "migration" threats, and in this paper we focus on the examination of cooperative (core) and noncooperative (Nash) notions of stability of jurisdiction structures.

In our model each agent incurs two types of costs. One is her contribution towards the cost of public project described above. The second is "transportation" cost, or disutility, steaming from the fact that the specific choice of public project made by the jurisdiction to which she belongs, in general, differs from her top choice. We further impose the efficiency principle that yields the project location that minimizes the total transportation cost of the jurisdiction. Under the linearity of transportation costs, the efficiency amounts to the median voter rule, where the project is placed at the location of the median agent in the jurisdiction. Since a median agent may not be uniquely determined, we have to specify the selection from the median set. In the first part of our analysis we avoid this problem by adopting the $M M$ rule that selects the mean of the two extreme medians of a given jurisdiction. We later relax this assumption by allowing a jurisdiction to place the project at any point of its median set.

The recent literature on cost sharing methods in this context contains two trends. One (Le Breton and Weber (2003), (2004), Haimanko et al. (2004a), Le Breton et al. (2004)) adopt the transferable utility framework and an unrestricted set of cost sharing allocations within every jurisdiction. However, in many situations the degree of freedom in selecting a redistribution scheme could be severely restricted by customs or law, and the side payments are virtually impossible. In this paper we follow the alternative approach (Alesina and Spolaore (1997), Casella (2001), Jehiel and Scotchmer (1997, 2001), Haimanko et al. (2004b)) 
who consider an Equal Share - ES scheme where all members of the same jurisdiction make an equal contribution towards the project cost. The interpretation is that agents are hold responsible for their preferences, and are not compensated for being away from the public project. Thus, agents located in the proximity of the public project incur a lower cost than those who are distant from the location of the public project.

The resolution of cost sharing mechanism and location of public project reduces the jurisdiction formation problem to a search for coalition structures that are stable under median and equal share rules. Note that under $M M$ and $E S$ rules our framework falls into the class of hedonic games (Banerjee et al. (2001) and Bogomolnaia and Jackson (2002)), where once a coalition is formed, one can uniquely determine the payoff of all its members. Hence, every agent forms well-defined preferences over possible jurisdictions she could be a member of. This framework allows us to consider both a cooperative stability notion (core), which is immune against deviations by any group of agents, and the non-cooperative Nash notion of stability, when only single agents can contemplate switching jurisdictions. Our results show that, in general, both core and Nash stable partitions may fail to exist. Both sets are, however, nonempty in the special case of equidistant societies, i.e, those with equal distances between every two adjacent agents.

One may argue that the lack of stable partitions is due to the rigidity of the hedonic framework where no jurisdiction can alter its pre-determined choice. Thus, we consider a quasi-hedonic modification of the game by allowing the project location at any of the jurisdiction medians. We demonstrate that the quasi-hedonic framework does not remove a possibility of empty core. However, by applying the technique of potential games (Rosenthal (1973) and Monderer and Shapley (1996)), we were able to demonstrate the existence of Nash stable partitions in this case.

Given the importance of agents' locations in our framework, we also investigate the existence of a stable jurisdiction structure which is stratified or consecutive, where, to recall, a jurisdiction is consecutive if for every two its members at different locations, all agents with "intermediate" locations belong to the same jurisdiction. It turns out that every Nash stable partition is consecutive. The situation, however, is more intricate regarding the core stability, and we show that a consecutive core stable partition may fail to exist even if the set of core stable partitions is nonempty. Moreover, there are non-consecutive core stable partitions even in equidistant societies. ${ }^{1}$

The paper is organized as follows. In the next section we present the model and definitions. Section 3 is devoted to the results on hedonic setting, whereas the quasi-hedonic framework is investigated in Section 4. We sketch the proofs and discuss the intuition of the results in Sections 3 and 4 whereas their formal proofs are relegated to the Appendix.

\section{The Model}

We consider a finite set $N=\{1, \ldots, n\}$ of agents. Each agent has symmetric singlepeaked preferences over the bounded interval $I$ of the real line $\mathbb{R}$. The single-peakedness

\footnotetext{
${ }^{1}$ This result is in contrast to Alesina and Spolaore (1997) who consider the continuous and uniform distribution of agents and rule out a possibility of formation of non-consecutive jurisdictions.
} 
and symmetry of preferences allow us to fully characterize an agent $i$ by her ideal point $l^{i}$ and we will refer to $l^{i}$ as the location of the agent $i$. Assume, without loss of generality, that $l^{1} \leq l^{2} \leq \cdots \leq l^{n}$.

The society $N$ can be partitioned into one or more jurisdictions. When formed, each jurisdiction has to choose a public project from $I$. The total cost of a public project is $g>0$, which is given exogenously and is independent of the composition of a jurisdiction. ${ }^{2}$ For simplicity, let us put $g=1$. We impose budget balancedness so that members of every jurisdiction finance the cost of their own project according to the chosen cost-sharing rule. Throughout the paper we assume that the cost is financed according to the Equal share (ES) rule, which requires the equal contribution $\frac{1}{|S|}$ of each member of jurisdiction $S$.

In addition to her contribution towards the financing of the public project, every jurisdiction member incurs a transportation cost, $d\left(l^{i}, l\right)$, between her own location and that of the public project at $l$. We assume that the transportation costs of all agents are linear; precisely, $d\left(l^{i}, l\right)=\left|l-l^{i}\right|$.

For every jurisdiction $S$ denote by $M(S)$ the set of its median project locations:

$$
M(S)=\left\{l \in S: \min \left[\left|\left\{i \in S \mid l^{i} \leq l\right\}\right|,\left|\left\{i \in S \mid l^{i} \geq l\right\}\right|\right] \geq \frac{1}{2}|S|\right\}
$$

That is, $l$ is a median location of the jurisdiction $S$ if, at least, half of the members of $S$ are located to the left of $l$ and at $l$, and, at least, half are located to the right of $l$ and at $l$ itself. It is easy to see that $M(S)$ is a nonempty interval. Under the assumption of linearity of transportation costs, every jurisdiction that minimizes its aggregate transportation cost, $D(S)$, must place the public project at one of its median locations. Since the median set $M(S)$ is not necessarily a singleton, we need to specify a selection rule from this set.

We first consider the case with the pre-determined selection rule by assuming that every jurisdiction $S$, if it forms, always chooses the mean $m(S)$ of two extreme points of the median set $M(S)$. Thus, $m(S)$ is equidistant from the endpoints of $M(S)$. (Obviously, if the set $M(S)$ consists of a single point, this very point will be chosen by $S$.) We will call this selection mechanism the $M M$ rule and examine it in the next section. We further relax the exogeneity of the selection rule and allow a jurisdiction to choose any alternative location from the median set $M(S)$.

It is important to note that the $E S$ and $M M$ rules define a hedonic game: once a jurisdiction is formed, the total cost for each its member is uniquely determined. Specifically, a member $i$ of jurisdiction $S$ incurs the total cost

$$
c_{i}(S)=\left|m(S)-l^{i}\right|+\frac{1}{|S|} .
$$

\footnotetext{
${ }^{2}$ This restriction can be easily relaxed by assuming that the cost of the public project is positively correlated with the size of the jurisdiction, namely, $g(S)=g+\alpha|S|$, where $g$ and $\alpha$ are positive parameters, and $|S|$ stands for the cardinality of the set $S$.
} 
Thus, every agent has well-defined preferences over the set of jurisdictions to which she could belong, and the cost of all agents is fully determined once we know the partition of the society into jurisdictions. Note that in this paper, the gross benefits derived from the consumption of the public good are not specified and are assumed only to be large enough to rule out the possibility that any agent would be left out of all jurisdictions that produce public projects. In order to guarantee this voluntary participation, it suffice to assume that benefits exceed the project cost.

We first consider the standard notion of core stability, where a partition $P$ is core stable if no group of agents (not necessarily from the same jurisdiction) could reduce their costs by creating their own jurisdiction. This notion is closely related to that of the core of a coalition structure (Aumann and Drèze (1974)):

Definition 2.1: Let $P=\left\{S_{1}, \ldots, S_{K}\right\}$ be a jurisdiction structure. We say that a jurisdiction $S \subset N$ blocks $P$ if

$$
c_{i}(S)<c_{i}\left(S_{k(i)}\right)
$$

for all $i \in S$, where $k(i)$ is the number of the jurisdiction in $P$ that contains $i$. A jurisdiction structure $P$ is called core stable if there exists no jurisdiction $S$ which blocks $P$.

The next definition is that of Nash stability, that can be viewed as a free mobility equilibrium, where no agent has an incentive to move to either another jurisdiction or to the "empty" one.

Definition 2.2: A jurisdiction structure $P=\left\{S_{1}, \ldots, S_{K}\right\}$ is called Nash stable if

$$
c_{i}\left(S^{i}\right) \leq g \quad \text { and } \quad c_{i}\left(S^{i}\right) \leq c_{i}\left(S_{k} \cup\{i\}\right)
$$

for every agent $i$ and all $S_{k} \in P$.

Note that a Nash stable jurisdiction structure is, in fact, a pure strategies Nash equilibrium of the non-cooperative game, where each agent announces her "address" and all the agents with the same address form a jurisdiction (Le Breton and Weber (2004)).

Since by Definition 2.2, an individual can contemplate move to another jurisdiction without the consent of its members, some or even all of them could be worse off after this move, which is impossible under the core stability. Thus, there is no logical connection between the notions of core and Nash stability.

We will examine both notions of stability and examine the existence of core and Nash stable jurisdiction structures. When a stable partition exists, we will investigate its stratification or consecutiveness properties (cf. Greenberg and Weber (1986)):

Definition 2.3: A jurisdiction $S \subset N$ is consecutive if for all $i, k \in S, l^{i}<l^{k}$, every agent $j$ with $l^{i}<l^{j}<l^{k}$ also belongs to $S$.

It would be useful to introduce an additional notation. For every jurisdiction $S$ we denote by $L(S)$ the minimal interval that contains locations of all members of $S$. Obviously, $L(S)$ is the convex hull of locations of all peripheral members of $S$. Denote by $\tilde{L}(S)$ the interior of $L(S)$. Then, according to Definition 2.2, a jurisdiction $S$ is consecutive if there is no agent $j \notin S$, whose location $l^{j}$ lies in the interior of $L(S)$, i.e. $l^{j} \in \tilde{L}(S)$. This interpretation of consecutiveness allows to introduce the notion of consecutive partition: 
Definition 2.4: A partition $P=\left\{S_{1}, \ldots, S_{K}\right\}$ is consecutive if for every two jurisdictions $S_{k}, S_{t} \in P, \quad S_{k} \neq S_{t}$, the intersection $\tilde{L}\left(S_{k}\right) \cap \tilde{L}\left(S_{t}\right)$ is empty.

Obviously, every jurisdiction in a consecutive partition is consecutive, whereas the opposite is not necessarily true. Indeed, consider the following society with four agents, 1,2,3,4, where $l^{1}=l^{2}<l^{3}=l^{4}$. By Definition 2.3, jurisdictions $S_{1}=\{1,3\}$ and $S_{2}=\{2,4\}$ are consecutive. However, the partition $\left\{S_{1}, S_{2}\right\}$ is not. Indeed, the intervals $L\left(S_{1}\right)$ and $L\left(S_{2}\right)$ are identical and have a common nonempty interior.

\section{Results on Hedonic Games}

Our first result indicates that there could be a society without core stable partitions:

Proposition 3.1: Under $E S$ and $M M$ rules, a core stable jurisdiction structure may fail to exist.

Consider a society with eight agents, located at the points $l^{1}=0, l^{2}=l^{3}=l^{4}=A=\frac{1}{42}$, $l^{5}=B=\frac{1}{14}=\frac{3}{42}$ and $l^{6}=l^{7}=l^{8}=C=\frac{11}{42}-\delta$, where $\delta$ is a small positive number (see Figure 1).

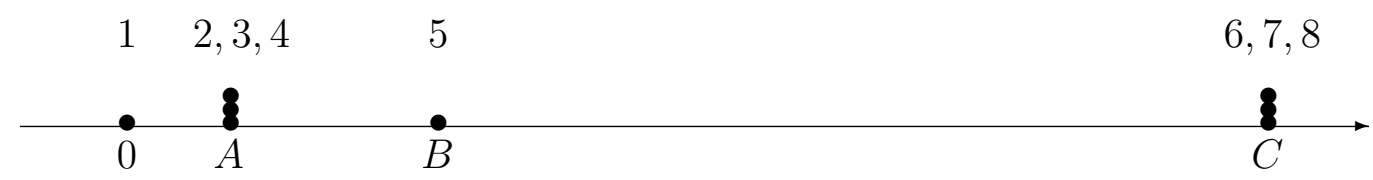

Figure 1. A society that admits no core stable jurisdiction structure under $E S$ and $M M$ rules.

The complete proof of the fact that this society does not admit a core stable jurisdictional structure, as well as of all other results of the paper, is relegated to the Appendix. Here we provide the sketch of the proof.

In this example there are three groups of agents: agent 1 at 0 on the left, agents $2,3,4,5$, located in the middle at points $A$ and $B$, and agents 6,7,8 located at $C$ on the right. The last three should be together in the same jurisdiction; moreover, they would prefer to be together with $2,3,4,5$ in the jurisdiction $N \backslash\{1\}$. But if $N \backslash\{1\}$ forms, then agent 1 , together with the group $6,7,8$ would form a blocking jurisdiction. Next, if $\{1,6,7,8\}$ forms, the agents $2,3,4,5$ would offer agent 1 to form a blocking jurisdiction $\{1,2,3,4,5\}$. But then, again, agents $6,7,8$ together with agents $2,3,4,5$ are better off in the jurisdiction $N \backslash\{1\}$. Thus, there is a blocking cycle of "dividing a dollar" type game, and since the grand jurisdiction $N$ is core stable, no core stable partition would emerge.

It is important to point out that a core stable jurisdiction structure $P$ does not need to be consecutive. Moreover, even when a particular society admits core stable jurisdiction structures, it could be the case that none of these stable structures is consecutive. 
Proposition 3.2: Under $E S$ and $M M$ rules, a consecutive core stable jurisdiction structure may fail to exist even if the set of core stable jurisdiction structures is nonempty.

Consider the following example with 34 agents, where $l^{1}=0, l^{2}=\cdots=l^{14}=a, l^{15}=l^{16}=$ $l^{17}=b, l^{18}=\cdots=l^{27}=c$, and $l^{28}=\cdots=l^{34}=d$ (see Figure 2). The exact values of $a, b, c, d$ are defined in the Appendix; one can, however, notice their relative values on Figure 2 , where $d \approx 1 / 4$. We claim that for this society, a set of core stable jurisdiction structures, while being nonempty, contains no consecutive partitions.

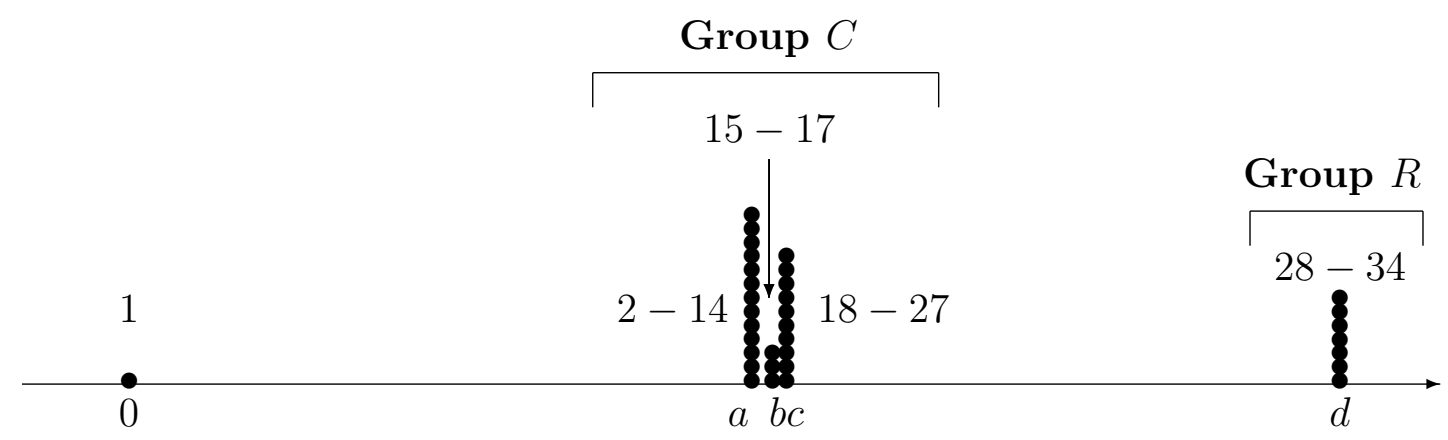

Figure 2. A society which admits a core stable partition, but with no consecutive core stable partitions.

There are three groups of agents: agent 1, located at the far left, a large group $C$ of 26 agents, located around the middle (but not at the same location), and a medium size group $R$ of 7 agents located on the right. The large group $C$ would not admit either of smaller groups, since such an admission would shift the median of $C$ and could make some of its members worse off. At the same time the group $R$ would accept agent 1, who, given increasing returns to scale, would be happy to join a larger group. Thus, there is a core stable partition which consists of two jurisdictions, the group in the middle $C$ and the union of two smaller groups $\{1\} \bigcup R$, while there are no consecutive core stable jurisdiction structures.

Even though a core stable structure may fail to exist, there are societies that do admit core stable (and even consecutive) jurisdiction structures. One such class is equidistant societies, where the distance between every two adjacent agents is the same. Formally, a society $N$ is equidistant if there exists $l>0$, such that $l^{i}-l^{i-1}=l$ for $i=2, \ldots, n$. We have

Proposition 3.3: Under $E S$ and $M M$ rules, every equidistant society admits a core stable consecutive jurisdiction structure.

However, even in this case, there could exist a non-consecutive core stable jurisdiction structure:

Proposition 3.4: There exist equidistant societies, that, under $E S$ and $M M$ rules, admit a core stable non-consecutive jurisdiction structure. 
It would be useful to introduce the notion of a peripheral agent for jurisdiction $S$, where an agent $i$ is peripheral for $S$ if her location $l^{i}$ is either leftmost or rightmost among all locations of members of $S$.

To show an example of an equidistant society with a core stable non-consecutive jurisdiction structure, consider eight agents $\{1, \ldots, 8\}$, and their two-jurisdiction partition $P=\{\{2,3,4,5\},\{1,6,7,8\}\}$. We choose the distance $l$ between adjacent agents in such a way that (i) the lowest cost of a peripheral agent in a consecutive jurisdiction of size $k$ is attained at $k=4$; and (ii) agent 1 prefers jurisdiction $\{1,6,7,8\}$ to staying alone. Then no group of agents can block, since in any jurisdiction, different from $\{1\}$, at least one agent, who is not 1 , is peripheral, and thus she would face a cost at least as high as in $P$.

Proposition 3.3 is, in fact, a finite counterpart of the Alesina and Spolaore (1997) result obtained for a continuum of agents uniformly distributed over the finite interval. However, unlike Alesina and Spolaore (1997), we consider a possibility of non-consecutive jurisdictions and show in Proposition 3.4 that a core stable partition can be non-consecutive. While the lack of consecutiveness in core stable structures may seem counterintuitive, the phenomenon of nonconsecutive jurisdiction formation has been noticed in Greenberg and Weber (1985) and observed in various political environments (Brams et al. (2002)).

Let us now turn to Nash stability. Contrary to core stable partitions, any Nash stable partition is stratified:

Proposition 3.5: Under $E S$ and $M M$ rules, every Nash stable jurisdiction structure is consecutive. Moreover, in every Nash stable partition, any two agents located at the same point belong to the same jurisdiction.

The intuition is clear. In a non-consecutive partition, there are two agents, $i \in S$ and $j \in S^{\prime}$ from different jurisdictions, such that $i$ is closer to $m\left(S^{\prime}\right)$ than $j$, whereas $j$ is closer to $m(S)$ than $i$. But if $i$ does not want to switch to $S^{\prime}$, then $j$ would have liked to move to $S$, implying that such a jurisdictional structure is not Nash stable.

In general, we cannot guarantee the Nash stability in our framework. The source for possible Nash instability is that by moving to another jurisdiction, an agent necessarily affects the recipient jurisdiction project's location, and, therefore, contributions of its members. This argument allows us to construct a "cycle of individual improvements" accompanied by other agents' cost increases. When the impact of a migrating agent on the project location in a new jurisdiction is mitigated (see next section), we obtain the Nash stability in the quasi-hedonic framework.

Proposition 3.6: Under $E S$ and $M M$ rules, a Nash stable jurisdiction structure may fail to exist.

Consider the following example with five agents, where $l^{1}=0, l^{2}=l^{3}=\frac{23}{30}, l^{4}=\frac{29}{30}$ and $l^{5}=\frac{191}{120}($ see Figure 3$)$. 


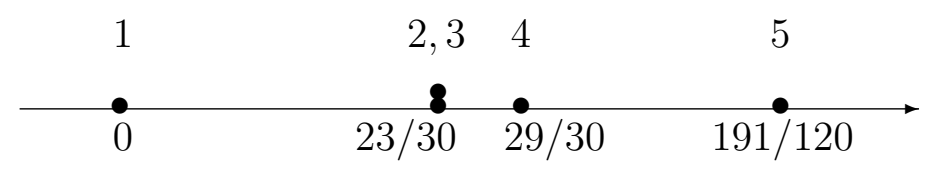

Figure 3. A society that admits no Nash stable jurisdiction structure.

We will show that, in a Nash stable partition, agents 2 and 3 belong to the same jurisdiction, and moreover, are joined by agent 4 . Given the formed group $T=\{2,3,4\}$, agent 1 will join $T$ only if agent 5 joins it, too. However, agent 5 would join $T$ only if agent 1 would not. These "cat and mouse" preferences rule out the existence of a pure strategies Nash equilibrium.

But, as in the core stability examination, the equal distances between every pair of adjacent agents guarantee Nash stability:

Proposition 3.7: Under $E S$ and $M M$ rules, every equidistant society admits a Nash stable jurisdiction structure (which, by Proposition 3.5, is consecutive).

We have already pointed out that there was no logical relationship between the concepts of stability and Nash stability. It is formally stated by the following proposition:

Proposition 3.8: Under $E S$ and $M M$ rules,

(i) there exist core stable jurisdiction structures which are not Nash stable;

(ii) there exist Nash stable jurisdiction structures which are not core stable.

In fact, we show that in the society examined in Figure 1 with no core stable partition, the grand jurisdiction is Nash stable. On the other hand, the society in Figure 3, which does not admit a Nash stable partition, has a core stable partition $P=\{\{1\},\{2,3,4\},\{5\}\}$.

\section{Quasi-hedonic games}

We now relax the $M M$ rule and allow a jurisdiction to choose any project from its median set. We call this requirement arbitrary median - $A M$ rule. The $E S$ and $A M$ rules create a quasi-hedonic setting, where the location of the public project and, hence, the transportation costs are not uniquely determined by the composition of the jurisdiction when its median set is not a singleton. To determine the agents' costs, one has to specify the pair $(P, L)$, where $P=\left\{S_{k}\right\}_{1 \leq k \leq K}$ is a partition of $N$, and the set $L=\left\{m_{1}, \ldots, m_{K}\right\}$ consists of project selections from the corresponding median sets, i.e., $m_{k} \in M\left(S_{k}\right)$. We denote by $c_{i}(m, S)$ the total cost of an agent $i \in S$ in this game when $S$ chooses the location $m$ :

$$
c_{i}(m, S)=\left|m-l^{i}\right|+\frac{1}{|S|}
$$

Definition 2.1 of core stability can be modified to the quasi-hedonic setting: 
Definition 4.1: Let $(P, L)$ be a pair, where $P=\left\{S_{1}, \ldots, S_{K}\right\}$ is a jurisdiction structure and $L=\left\{m_{1}, \ldots, m_{K}\right\}$ is a set of locations with $m_{k} \in M\left(S_{k}\right)$ for all $k=1, \ldots, K$. We say that a jurisdiction $S \subset N$ blocks $(P, L)$ via $m$ if for all $i \in S$

$$
c_{i}(m, S)<c_{i}\left(m_{k(i)}, S_{k(i)}\right),
$$

where $k(i)$ is the number of the jurisdiction in $P$ to which $i$ belongs.

A partition $(P, L)$ is called core stable if there is no jurisdiction $S$ and location $m \in$ $M(S)$ such that $S$ blocks $(P, L)$ via $m$.

Alas, as the following proposition shows, our extension of the set of possible project choices for all jurisdictions does not guarantee the existence of a core stable jurisdiction structure:

Proposition 4.2: Under $E S$ and $A M$ rules, a core stable jurisdiction structure may fail to exist.

Consider a society with five agents, $N=\{1,2,3,4,5\}$, whose locations are given by $l^{1}=$ $l^{2}=l^{3}=0, l^{4}=l^{5}=\frac{19}{60}($ see Figure 4$)$.

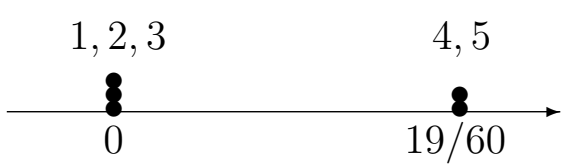

Figure 4. A society that admits no core stable jurisdiction structure under $E S$ and $A M$ rules.

The essence of this example, as that in Proposition 3.1 in the hedonic case, is again the same as in the "dividing a dollar" cooperative game. There are three groups of agents, $\{1\}$, $\{2,3\},\{4,5\}$, which "bribe" each other in the cyclical manner. Indeed, suppose, that the first two groups cooperate by forming the jurisdiction $\{1,2,3\}$. Then, the group $\{4,5\}$ offers $\{2,3\}$ to cooperate and locate the project at the point, say, $\frac{9}{120}$, where all agents $1,2,3,4$ are better off.

But, facing the jurisdiction $\{2,3,4,5\}$, agent 1 would offer the cooperation to the members of the last group, $\{4,5\}$. This again increases the payoff of all three agents $1,4,5$. And finally, the group $\{2,3\}$ offers 1 to join them again: the group $\{1,2,3\}$ guarantees all its members a higher payoff. At the same time, the union of all groups, $\{1,2,3,4,5\}$ is unstable, as the jurisdiction $\{4,5\}$ would block it.

In order to define Nash stability for quasi-hedonic games, we have to specify a median shift in the jurisdiction joined by a new member. Note that for every jurisdiction $S$ and every agent $i \notin S$, the relationship between the median sets of $S$ and $S \bigcup\{i\}$ is given by the following: 
Remark 4.3: For every jurisdiction $S$ and every agent $i \notin S$, the intersection of two sets, $M(S)$ and $M(S \bigcup\{i\})$, is a singleton.

In this setup we minimize the impact of a new member on the jurisdiction's choice of public project. Suppose agent $i \in T$ joins jurisdiction $S$ that has selected public project $m \in M(S)$. If $m$ is a median of the enlarged coalition $S^{\prime}=S \bigcup\{i\}$ as well, then $m$ will naturally be sustained by $S^{\prime}$. If $m$ is not a median of $S^{\prime}$ then, by Remark 4.3, there is a unique project $m^{\prime}$ which is the median of both $S$ and $S^{\prime}$. In order to preserve consistency of the median choice by both $S$ and $S^{\prime}, m^{\prime}$ will be chosen by $S^{\prime}$. The similar procedure will be applied to the choice of the median in the jurisdiction $T$ abandoned by agent $i$. We will call this selection mechanism by consistent median - $C M$ rule.

Definition 4.4: Let $(P, L)$ be a pair, where $P=\left\{S_{1}, \ldots, S_{K}\right\}$ is a jurisdiction structure and $L=\left\{m_{1}, \ldots, m_{K}\right\}$ is a set of locations with $m_{k} \in M\left(S_{k}\right)$ for all $k=1, \ldots, K$. We say that $(P, L)$ is Nash stable if, for any agent $i$ and for any jurisdiction $S_{k} \in P$ such that $i \notin S_{k}$, we have

$$
c_{i}\left(m_{k(i)}, S_{k(i)}\right) \leq g \quad \text { and } \quad c_{i}\left(m_{k(i)}, S_{k(i)}\right) \leq c_{i}\left(M\left(S_{k}\right) \cap M\left(S_{k} \bigcup\{i\}\right), S_{k} \bigcup\{i\}\right)
$$

It turns out that the $C M$ rule which allows flexibility of jurisdictions' project choices brings about the existence of a Nash stable partition:

Proposition 4.5: Under $E S$ and $C M$ rules, there exists a Nash stable partition.

To prove this result we use the potential functions approach pioneered by Rosenthal (1973), and further developed by Monderer and Shapley (1996) and Konishi et al. (1997, 1998). In order to apply this technique in our framework, we define the function $\pi$ over the set $\mathcal{P}$ of all partitions of $N$ and then show that every minimum of $\pi$ yields a pure strategies equilibrium of the quasi-hedonic game.

To conclude, consider again the society in Figure 3 (Proposition 3.6), where a Nash stable partition fails to exist under $E S$ and $M M$ rules. Consider, however, the partition $\{\{1\},\{2,3,4\},\{5\}\}$. Under $E S$ and $C M$ rules, agent 5 would not join the jurisdiction $\{2,3,4\}$. If she were to join, the location of the public project in the enlarged coalition would remain at the point $\frac{23}{30}$ and she would rather stay alone. Thus this partition is Nash stable under $E S$ and $C M$ rules.

\section{Appendix}

Proof of Proposition 3.1: Consider the society presented by Figure 1 and denote $X=$ $\{2,3,4\}, Y=\{6,7,8\}$. Suppose, in negation, that there is a stable partition $P$. 
Note first, that partition $P$ cannot assign every member of $Y$ the total cost of more than $\frac{1}{3}$, since otherwise $Y$ would block $P$. We proceed in several steps:

Step 1. Suppose that there is a jurisdiction $S \in P$ such that $|S \bigcap Y|=1$. Then $i \in Y \bigcap S$ contributes more than $\frac{1}{3}$. Indeed, if $|S| \leq 2, i$ pays more than $\frac{1}{3}$. If $|S| \geq 3$ then $m(S) \leq B$ and $i$ contributes at least $\frac{1}{6}+(C-B)=\frac{7}{42}+\frac{8}{42}-\delta=\frac{15}{42}-\delta>\frac{15}{45}=\frac{1}{3}$.

Step 2. Suppose that there is a jurisdiction $S^{\prime} \in P$ such that $\left|S^{\prime}\right|>3$ and $\left|S^{\prime} \cap Y\right|=2$. Then both $j, k, \in Y \cap S^{\prime}, j \neq k$, contribute more than $\frac{1}{3}$. Indeed:

If $\left|S^{\prime}\right|=7$, then $j$ and $k$ contribute each $\frac{1}{7}+C-A=\frac{1}{7}+\frac{10}{42}-\delta>\frac{1}{3}$.

If $4<\left|S^{\prime}\right|<7$, then $m\left(S^{\prime}\right) \leq B$, and $j$ and $k$ contribute each at least $\frac{1}{6}+C-B>\frac{1}{3}$.

If $\left|S^{\prime}\right|=4$, then $j$ and $k$ pay each at least $\frac{1}{4}+\frac{C-B}{2}=\frac{1}{4}+\frac{4}{42}-\frac{\delta}{2}>\frac{1}{3}$.

Step 3. All agents in $Y$ stay in the same jurisdiction in $P$. If the members of $Y$ are split among three jurisdictions, then each member of $Y$ contributes more than $\frac{1}{3}$, and $P$ is blocked by $Y$. If the members of $Y$ are split among two jurisdictions, $S$ and $S^{\prime}$, then $\left|S^{\prime} \cap Y\right|=2$ and $|S \cap Y|=1$. If either $\left|S^{\prime}\right|>3$ or $\left|S^{\prime}\right|=2$, then, according to Steps 1 and 2 , all agents from $Y$ contribute more than $\frac{1}{3}$, which is impossible.

Hence, we must have $\left|S^{\prime}\right|=3$, yielding $m\left(S^{\prime}\right)=C$. But in this case the jurisdiction $S^{\prime} \cup Y$ would block $P$.

Thus, there is a jurisdiction $T \in P$ such that $Y \subset T$.

Step 4. Then $|T|>3$, i.e, $T \neq Y$. Indeed, if $T=Y$ then contributions of members of $T$ equal to $\frac{1}{3}$, and contributions of other agents are not lower than $\frac{1}{5}$, for there are only 5 other agents. Now, observe that $S^{1}=Y \bigcup X \bigcup\{5\}$ blocks $P$, as the contributions of members of $Y$ and $X$ at $S^{1}$ are $\frac{1}{7}+C-B$ and $\frac{1}{7}+B-A$, being lower than their contributions at $P$, which are $\frac{1}{3}$ and $\frac{1}{5}$, respectively.

Step 5. $|T| \geq 7$. Indeed, if $4 \leq|T| \leq 6$, the contributions of agents from $N \backslash T$ are not lower than $\frac{1}{4}>\frac{2}{42}+\frac{1}{5}$, whereas $m(T) \geq \frac{\bar{B}+C}{2} \geq \frac{7}{42}-\frac{\delta}{2}$, hence, contributions of members of $T \backslash Y$ are not lower than $\frac{1}{6}+\frac{4}{42}-\frac{\delta}{2}>\frac{2}{42}+\frac{1}{5}$; this means that the coalition $N \backslash Y$ would block $P$, for members of this coalition contribute not higher than $\frac{2}{42}+\frac{1}{5}$.

Step 6. $|T| \neq 7$. Indeed, let jurisdiction $|T|$ contain 7 members and consider an agent $i \in N \backslash T$. Since contributions of members of $Y$ in $T$ are not lower than $\frac{1}{7}+C-B=\frac{1}{3}-\delta>\frac{1}{4}$, and the contribution of $i$ in $P$ is equal to 1 , it follows that $Y \bigcup\{i\}$ blocks $P$.

Step 7. $T$ is not the grand jurisdiction. Indeed, since $\frac{1}{8}+C-B+\frac{B-A}{2}>\frac{1}{3}$, the grand jurisdiction $N$ is blocked by $Y$.

Therefore, a stable partition does not exist.

Proof of Proposition 3.2: Denote $N^{a}=\{2, \ldots, 14\}, N^{b}=\{15,16,17\}, N^{c}=$ 
$\{18, \ldots, 27\}, R=\{28, \ldots, 34\}$, and $C=N^{a} \cup N^{b} \cup N^{c}$ ( $R$ for "right", and $C$ for "center".) The locations $a, b, c, d$ obtain the following values:

$$
\begin{gathered}
a=\frac{1}{8} ; \quad b=\frac{1}{8}+\frac{2}{26 \cdot 27}+2 \varepsilon ; \\
c=\frac{1}{8}+\frac{2}{26 \cdot 27}+\frac{2}{33 \cdot 34}+2 \varepsilon+2 \delta ; \\
d=\frac{1}{8}+\frac{2}{26 \cdot 27}+\frac{2}{33 \cdot 34}+\frac{26}{33 \cdot 7}+2 \varepsilon+2 \delta-\xi,
\end{gathered}
$$

where $\varepsilon, \delta$ and $\xi$ are very small positive numbers. We shall show that the set of core stable partitions, while nonempty, does not contain a consecutive partition.

We will utilize the following four observations:

(i) $\frac{1}{8}<\frac{13}{33 \cdot 7}+\frac{1}{14}<\frac{1}{33}+d-c<\frac{1}{7}<\frac{1}{34}+d-c+\frac{c-b}{2}$. This implies that all members of group $R$ have the following preferences:

$$
R \bigcup Q \succ R \bigcup Q^{\prime} \succ R \bigcup C \succ R \succ R \bigcup C \bigcup\{1\}
$$

where $Q$ is a nonempty jurisdiction with fewer than 7 agents, and $Q^{\prime}$ is a jurisdiction with exactly 7 agents. That is, all agents located at $d$, would prefer to be joined by at least one, but no more than six, other agents, to being in the jurisdiction with 7 other agents; the latter outcome is preferred to being in the jurisdiction with all other agents, excluding 1. This, in turn, is preferable to forming jurisdiction $R$, whereas the grand coalition is the least desired option among those listed here.

(ii) $\frac{1}{33}+c-a<\frac{1}{27}$. This implies that all members of $C$ prefer $C \bigcup R$ to both $C \bigcup\{1\}$ and $C$.

(iii) $\frac{1}{26}+c-b+\frac{b-a}{2}<\frac{1}{23}$. This implies that all members of $C$ prefer $C$ to participating in a jurisdiction with no more than 23 agents.

(iv) $\frac{1}{26}<\frac{1}{27}+\frac{b-a}{2}$. The members of $N^{c}$ prefer $C$ to $C \bigcup\{1\}$, and thus, would be worse off when agent 1 joins $C$.

We first show that the partition $\bar{P}$ into two jurisdictions, $C$ and $\{1\} \cup R$, is core stable.

Observation (i) implies that no member of group $R$ would engage in blocking within the jurisdiction whose median is to the left of $d$. Thus, a jurisdiction $S$, that contains a member of $R$, could block only if its median $m(S)$ is located at $d$, implying that $|S|<14$. However, observation (iii) guarantees that no member of $C$ would find it profitable.

Thus, it remains to consider possible blocking threats to $\bar{P}$ from jurisdictions $S \subset$ $\{1\} \bigcup C$. Observation (iv) implies that $\{1\} \bigcup C$ itself cannot block. The case $S=\{1\}$ is, obviously, impossible; hence, $S$ contains some of agents from $C$. Observations (iii) and (iv) imply that $23 \leq|S| \leq 26$ and $S$ contains both types of agents in $C$, those are located to the left and those are located to the right of $m(C)=\frac{a+b}{2}$. Since the contribution to finance a project in $S$ is at least as much as in $C$, those agents from $S$ to whom $m(S)$ is not closer than $m(C)$ would reject the membership in $S$, a contradiction which shows that $\bar{P}$ is indeed, 
core stable.

Now assume, in negation, that there is a consecutive core stable partition $P$.

First, consider the case where there is $S \in P$ that contains $C$.

If $S \cap R=\emptyset$, then $P$ is either $\{\{1\}, C, R\}$ or $\{\{1\} \bigcup C, R\}$. But both would be blocked by $C \bigcup R$, as by observation (i), group $R$ prefers $C \bigcup R$ to $R$, and by observation (ii), $C$ prefers $C \bigcup R$ to both $C \bigcup\{1\}$ and $C$.

If $S \cap R \neq \emptyset$, then $S$ does not contain agent 1 (otherwise $m(S) \leq m(N)$, the grand coalition, and by observation (i), $R$ would block $P$ ). But then $\{1\} \bigcup R$ blocks $P$.

Consider now the case where $C$ is not a subset of any jurisdiction from $P$. Observation (iii) implies that if all jurisdictions in $P$ contain no more than 23 agents, $C$ would block $P$. Hence, there is a jurisdiction $S \in P$ with $|S| \geq 24$. Obviously, $m(S) \leq c$, because no more than 7 agents from $S$ are located to the right of $c$.

Let $S \cap R \neq \emptyset$. Then, $|S| \geq 33$, since otherwise $1 /|S|+26 /(33 \cdot 7)-\xi>1 / 7$ and $R$ will block $P$. But if $|S| \geq 33$, then consecutiveness implies that $C \subset S$, a contradiction.

Let $S \cap R=\emptyset$. Since $S$ is consecutive, the group $(\{1\} \cup C) \backslash S$ contains two (possibly empty) consecutive groups of agents, denoted by $X_{l}$ and $X_{r}\left(X_{l}\right.$ is to the left of $\left.X_{R}\right)$.

We claim that all the agents in $R$ belong to the same jurisdiction $T$. Indeed, if it is not the case then either there exists a (unique, due to consecutiveness!) jurisdiction $Q \in P$ with $Q \backslash R \neq \emptyset$, and then $R \cup Q$ will block $P$, or not, in which case $R$ itself will block $P$.

We now have three cases:

Case 1. $\left|X_{l}\right|=3$ (hence, $X_{r}=\emptyset$ ). In this case each of two agents from $X_{l} \backslash\{1\}$ will be better off by joining $S$. The same holds for every member of $S$, since $m(S \bigcup\{i\})=m(S)=b$, where $i \in X_{l} \backslash\{1\}$. Hence, $P$ is blocked by a coalition $S \bigcup\{i\}$.

Case 2. $0<\left|X_{l}\right|<3$. Then, an agent 1 contributes more than $\frac{1}{2}$. At the same time if she joins a jurisdiction $T$, her contribution would be not higher then $\frac{1}{8}+d<\frac{1}{2}$, and, again, her migration does not affect $m(T)$. Hence, $T \bigcup\{1\}$ blocks $P$.

Case 3. $X_{l}=\emptyset$. Then, $X_{r} \neq \emptyset$, since otherwise $S$ contains $C$, the case which has been already covered. Moreover, we have $m(S)=a$. If a member of $X_{r}$ migrates to jurisdiction $S$, she would pay less than in $P$ (where her contribution is higher than $\left.\frac{1}{10}\right)$ ). Since $m(S)=m(S \bigcup\{i\})=a$, it follows that $S \bigcup\{i\}$ is a blocking coalition.

Therefore, there is no consecutive core stable partition.

Proof of Proposition 3.3: Consider an equidistant society where the distance between any two adjacent agents is $l>0$. Note that in any consecutive jurisdiction with $k \leq n$ agents the total contribution of the peripheral agent is given by $f_{l}(k)$, where

$$
f_{l}(k)=\frac{1}{k}+\frac{k-1}{2} l .
$$

We extend the domain of the function $f_{l}$ to the set of all positive real numbers, so that the 
function $f: \mathbb{R}_{++} \rightarrow \mathbb{R}$ is defined by:

$$
f_{l}(x)=\frac{1}{x}+\frac{x-1}{2} l
$$

Note that $f_{l}(\cdot)$ is convex, attains its minimum at $x^{*}=\sqrt{2 / l}$, is decreasing on the interval $\left(0, x^{*}\right)$ and is increasing on the interval $\left(x^{*}, \infty\right)$. Thus, we obtain the existence of an integer $k^{*}$ such that $f(k) \geq f\left(k^{*}\right)$ for all positive integers $k$ (if there are two such $k^{*}$, we choose a large one among the two). This value $k^{*}$ indicates the (optimal) size of a consecutive jurisdictions that minimizes the cost of the peripheral agents.

If $k^{*} \geq n$, we claim that the grand jurisdiction $N$ is stable. Suppose, in negation, that there is a jurisdiction $S$ that blocks $N$. Then the contribution of the peripheral agent $i$ in $S$ is, at least, $f_{l}(|S|)$. However, the contribution of $i$ in $N$ would be no more than $f_{l}(n)$. The convexity of $f$ implies $f_{l}(|S|) \geq f_{l}(n) \geq f_{l}\left(k^{*}\right)$, a contradiction to the fact that coalition $S$ blocks $N$.

Consider the case where $k^{*}<n$. Construct a consecutive partition $P$, where, starting with agent 1 , all agents are divided into $m$ consecutive jurisdictions of the size $k^{*}$, and, possibly, one jurisdiction $Q=\left\{m k^{*}+1, \ldots, n\right\}$ that consists of fewer than $k^{*}$ agents. We shall show that $P$ is stable. Assume, to the contrary, that there exists a blocking jurisdiction $S$ and consider two peripheral agents $i, j$ in $S$. Each of them contributes at least $f_{l}(|S|)$ in $S$. On the other hand, the maximal contribution of an agent in $N \backslash Q$ is $f_{l}\left(k^{*}\right)$. Since $f_{l}(|S|) \geq f_{l}\left(k^{*}\right)$, and $S$ is a blocking jurisdiction, it follows that neither $i$ nor $j$ belongs to $N \backslash Q$. Thus, $S$ is a subset of $R$ with $|S|<|R|$. But the contribution of $i$ (and $j$ ) in $R$ does not exceed $f_{l}(|Q|)$. However, the monotonicity of the function $f_{l}$ to the left of $k^{*}$ implies $f_{l}(|S|)>f_{l}(|Q|) \geq f_{l}\left(k^{*}\right)$, which contradicts the assumption of $S$ being a blocking jurisdiction.

Proof of Proposition 3.4: Consider an equidistant society with eight agents, where the distance between adjacent agents is $l=\frac{1}{8}$. It is easy to verify that that minimum of the function $f_{1 / 8}(\cdot)$, defined by $(11)$, is $\frac{7}{16}$ and is attained at $k^{*}=4$. Consider now the partition $P=\{\{2,3,4,5\},\{1,6,7,8\}\}$, at which every agent, except 1 , pays at most $\frac{7}{16}$. But in every jurisdiction $S$ with at least two agents, the peripheral agents pay at least that amount, and there is a peripheral agent (not agent 1 ), who will not take part in blocking via $S$. As for singletons, even $S=\{1\}$ does not block $P$, since the total contribution of 1 in $P$ is $\frac{5.5}{8}+\frac{1}{4}<1$. Thus, $P$ is core stable.

Proof of Proposition 3.5: Let $P$ be a Nash stable partition. We first demonstrate that agents in the same location belong to the same jurisdiction in $P$. Indeed, if there are two agents $i \in S^{i} \in P$ and $j \in S^{j} \in P$ with $l^{i}=l^{j}$, but $S^{i} \neq S^{j}$, take the one, say, $i$, whose total contribution, $c_{i}$, is greater or equal to that of $j, c_{j}$. The move of $i$ to $S^{j}$ is beneficial to agent $j$, both in terms of transportation costs to the median of $S_{j} \bigcup\{i\}$ and the monetary contribution to financing the public project in a larger jurisdiction. Thus, the 
total contributions of $i$ and $j$ in $S_{j} \bigcup\{i\}$, denoted $c_{i}^{\prime}$ and $c_{j}^{\prime}$, respectively, satisfy

$$
c_{i} \geq c_{j}>c_{j}^{\prime}=c_{i}^{\prime}
$$

a contradiction to the fact that $P$ is Nash stable. But once this is a case, it is obvious that consecutiveness of $P$ is guaranteed by consecutiveness of all the jurisdictions in $P$. Suppose by contradiction that there exist three agents, $i, j, k$ with $l^{i}<l^{j}<l^{k}$, such that $i, k \in S \in P$ whereas $j \in S^{\prime} \in P$ with $S \neq S^{\prime}$.

The Nash stability conditions applied for agents $i$ and $k$ imply

$$
\left|l^{i}-m(S)\right|+\frac{1}{|S|} \leq\left|l^{i}-m\left(S^{\prime} \bigcup\{i\}\right)\right|+\frac{1}{\left|S^{\prime}\right|+1}<\left|l^{i}-m\left(S^{\prime}\right)\right|+\frac{1}{\left|S^{\prime}\right|},
$$

or

$$
\left|l^{i}-m(S)\right|-\left|l^{i}-m\left(S^{\prime}\right)\right|<\frac{1}{\left|S^{\prime}\right|}-\frac{1}{|S|}
$$

Similarly,

$$
\left|l^{k}-m(S)\right|-\left|l^{k}-m\left(S^{\prime}\right)\right|<\frac{1}{\left|S^{\prime}\right|}-\frac{1}{|S|} .
$$

Since the function $|l-m(S)|-\left|l-m\left(S^{\prime}\right)\right|$ is monotone in $l$, it follows that

$$
\left|l^{j}-m(S)\right|+\frac{1}{|S|}<\left|l^{j}-m\left(S^{\prime}\right)\right|+\frac{1}{\left|S^{\prime}\right|}
$$

However, the last inequality implies

$$
\left|l^{j}-m(S \bigcup\{j\})\right|+\frac{1}{|S|+1}<\left|l^{j}-m\left(S^{\prime}\right)\right|+\frac{1}{\left|S^{\prime}\right|},
$$

a violation of the Nash stability requirement for $j$.

Proof of Proposition 3.6: Assume, in negation, that there exists a Nash stable partition $P$ for the society depicted on Figure 3. By Proposition 3.5, $P$ is consecutive, and agents 2 and 3 belong to the same jurisdiction. 
Let us show first that agent 4 will always join 2 and 3 . Indeed, if $S$ is a jurisdiction containing 2,3 , then $\frac{23}{30} \leq m(S)<\frac{29}{30}$. Thus, by joining 2,3 , agent 4 would contribute at most $\frac{1}{3}+\frac{1}{5}=\frac{8}{15}$, which is less than $\frac{13}{16}$, her contribution in the jurisdiction $\{4,5\}, \frac{59}{60}$, and 1 , her stand alone contribution.

Denote $T=\{2,3,4\}$ and consider the options available to agents 1 and 5 .

(i) Agent 1 joins $T$ only if agent 5 joins it as well. Indeed, the contribution of agent 1 at $N$ is $\frac{1}{5}+\frac{23}{30}=\frac{29}{30}<1$. However, her contribution at $T \bigcup\{1\}$ is $\frac{1}{4}+\frac{23}{30}=\frac{61}{60}>1$.

(ii) Agent 5 joins $T$ only if agent 1 does not. Indeed, the contribution of agent 5 at $N$ is $\frac{1}{5}+\frac{33}{40}=\frac{41}{40}>1$. However, her contribution at $T \bigcup\{5\}$ is $\frac{1}{4}+\frac{29}{40}=\frac{39}{40}<1$.

(i) and (ii) imply that there is no Nash stable partition. $\square$

Proof of Proposition 3.7: The proof proceeds in a sequence of claims. First, we establish the bounds for the size of the optimal jurisdiction $k^{*}$ that minimizes the contribution of the peripheral members given by equation (10).

Claim 1:

$$
\hat{k}<k^{*} \leq \hat{k}+1,
$$

where $\hat{k}$ is the (unique) positive solution of the equation

$$
\hat{k}(\hat{k}+1)=\frac{2}{l}
$$

Proof: Since the function $f$ is strictly decreasing to the left of its minimum and increasing to the right of it, it is easy to see that the minimal value of $f$ across all positive integers lies within the interval $(\hat{k}, \hat{k}+1]$, where $f_{l}(\hat{k})=f_{l}(\hat{k}+1)$. (The fact that the left side of the interval is open is due to the convention to take the largest value of $k$ if there are two minimizing values.) But the latter equality implies (18).

Claim 2: Let $P$ be a consecutive partition of $N$ into jurisdictions of sizes $r$ and $r+1$, where $k^{*} \leq r$. Then no agent would switch to another jurisdiction in $P$.

Proof: Take an agent $i \in S \in P$. Her contribution in $S$ does not exceed $f_{l}(|S|)$. If she joins another jurisdiction $T \in P$ then $i$ would be peripheral in $T \bigcup\{i\}$ and her contribution would be at least $f_{l}(|T|+1)$. But since $|S| \geq k^{*}$, we have $f_{l}(|T|+1) \geq f_{l}(|S|) \geq f_{l}\left(k^{*}\right)$. Thus, agent $i$ would not move to another existing jurisdiction in $P$.

Claim 3: A jurisdiction $S$ contains an agent who would rather stay alone if and only if $|S|>\frac{2}{l}$.

Proof: For every jurisdiction $S$, the incentive to leave and form a one-agent jurisdiction is strongest for the peripheral members of $S$. Their contribution is $f_{l}(|S|)=\frac{1}{|S|}+\frac{|S|-1}{2} l$, and they would prefer staying in $S$ rather than being alone if the last expression does not exceed 1 , or $|S| \leq \frac{2}{l}$.

Claim 4: If $n \geq m(m-1)$ for some positive integer $m$, there exists a consecutive partition of $N$ into consecutive jurisdictions with $m$ or $m+1$ members.

Proof: Let $Z=m(m-1)$. If $n=Z$, we divide all individuals into $m-1$ jurisdictions of the size $m$. For all numbers exceeding $Z$, we set the following process: 
(i) for $n=Z+1, \ldots, Z+m-1$ we add one agent to (different) existing jurisdictions;

(ii) for $n=Z+m$ we create $m$ jurisdictions of the size $m$;

(iii) for $n=Z+1+m, \ldots, Z+2 m-1$, we repeat (i);

(iv) for $n=Z+2 m$ we create $m+1$ jurisdictions of the size $m$; etc.

By repeating this process we can construct a required partition for every number $n$ of agents that is not less than $Z$.

We are now in position to prove the assertion of the proposition. By Claim 2, the grand jurisdiction is Nash stable whenever $n \leq \frac{2}{l}$. It remains to consider the case where $n>\frac{2}{l}$. By (19), we have

$$
n>\frac{2}{l}=\hat{k}(\hat{k}+1)
$$

and by Claim 1 ,

$$
n>\hat{k}(\hat{k}+1) \geq k^{*}\left(k^{*}-1\right) .
$$

Then by Claim 4 , we have a partition of $N$ into jurisdictions of sizes $k^{*}$ and $k^{*}+1$. Thus, by Claims 2 and 3, the proof is completed if $k^{*}+1 \leq \frac{2}{l}$. It remains, therefore, to consider the case where $k^{*}+1>\frac{2}{l}$. If the last inequality is satisfied then

$$
\hat{k}=-\frac{1}{2}+\sqrt{\frac{1}{4}+\frac{2}{l}}>\frac{2}{l}-2 .
$$

This inequality holds if and only if either

$$
\frac{2}{l}-\frac{3}{2}<0 \Leftrightarrow l>\frac{4}{3}
$$

or $l \leq \frac{4}{3}$ and

$$
\frac{1}{4}+\frac{2}{l}>\frac{4}{l^{2}}-3 \frac{2}{l}+\frac{9}{4} \Leftrightarrow 2-\sqrt{2} \leq \frac{2}{l} \leq 2+\sqrt{2} .
$$

Finally, combining these two cases, we obtain that the inequality $(22)$ is equivalent to $l \geq$ $2-\sqrt{2}$.

Then, either $l \geq 1$ or $2-\sqrt{2} \leq l<1$. In the first case the partition of $N$ into singletons is Nash stable. In the second case, if $n$ is even, then the partition of $N$ into consecutive pairs 
is Nash stable. If $n$ is odd, we claim that a partition of $N$ into consecutive pairs and one singleton is Nash stable. Indeed, since $f_{l}(x)$ attains its minimum at

$$
\sqrt{2 / l} \leq \sqrt{2+\sqrt{2}}<2
$$

for $l \geq 2-\sqrt{2}$, it follows that $k^{*}=2$, and no member of a two-agent jurisdiction wants to join another pair. Since $l<1$, no one wants to leave a two-person jurisdiction, and the inequality $k^{*}+1>\frac{2}{l}$ implies that being a singleton is preferable over being a member of a three-agent jurisdiction.

Proof of Proposition 3.8: Consider a society with eight agents, introduced in Figure 1 , where the agents are located on the interval $\left[0, \frac{11}{42}-\delta\right]$, where $\delta$ is a very small positive number, with the median of the grand jurisdiction located at $\frac{1}{21}$. It is easy to verify that the grand jurisdiction $N$ is Nash stable, whereas Proposition 3.1 demonstrates that the set of core stable partitions in this society is empty.

On the other hand, consider a society with five agents given by Figure 3, that was examined in the proof of Proposition 3.6, where the location of the agents are given by $l^{1}=0, l^{2}=l^{3}=\frac{23}{30}, l^{4}=\frac{29}{30}$ and $l^{5}=\frac{191}{120}$. We have proved that this society does not admit a Nash stable partition. Let us show, however, that the partition $P=\{\{1\},\{2,3,4\},\{5\}\}$ is core stable.

Suppose, in negation, that there exists a blocking jurisdiction $S$. Assume that agent 1 belongs to $S$. If $m(S)<\frac{23}{30}$, then $|S|=2$ and no other agent would join 1 in such a jurisdiction. If $m(S) \geq \frac{23}{30}$, agent 1 would join only if $S=N$ (otherwise her costs are at least $\left.\frac{1}{4}+\frac{23}{30}>1\right)$. However, agent 5 would not join $N$. Hence, $S$ does not contain 1 .

Note that agents 2 and 3 could contemplate joining $S$ only if $|S| \geq 4$. But at $\{2,3,4,5\}$ they contribute $\frac{1}{10}+\frac{1}{4}$ which exceeds $\frac{1}{3}$, the contribution of 2 and 3 in $P$. Hence, $S$ does not contain either of agents 2 and 3.

Thus, it remains to consider only two possibilities $S=\{4,5\}$ and $S=\{4\}$. It is trivial to check that neither of these jurisdictions can block $P$. Thus, $P$ is indeed core stable. $\square$

Proof of Proposition 4.2: Let us make few observations:

(i) The grand jurisdiction is not stable. Indeed, in this case, agents 4 and 5 pay $\frac{1}{5}+\frac{19}{60}=\frac{31}{60}$, while in a two-person jurisdiction their contribution would be smaller, namely, $\frac{1}{2}$.

(ii) No two agents stay separately alone as they would be better off by staying together. Indeed, say, in the jurisdiction $\{1,4\}$ each agent pays $\frac{1}{2}+\frac{19}{120}<1$.

(iii) No three-jurisdiction partition is stable. Indeed, by (ii), a partition into one triple and two singletons is not stable. However, given a partition into one singleton and two pairs, agents 1,2,3 would be better off by staying together.

(iv) No partition that contains a singleton, is stable. Indeed, since 4 and 5 contribute $\frac{31}{60}$ in the grand jurisdiction, none of them would stay alone and contribute 1 . Consider a partition $\{1\} \bigcup\{2,3,4,5\}$, where the second group chooses the location $r, 0 \leq r \leq \frac{19}{60}$. The 
contribution of agents 2 and 3 is $\frac{1}{4}+r$, and since 1,2,3 can form the jurisdiction together and contribute $\frac{1}{3}$, we have the inequality $\frac{1}{4}+r \leq \frac{1}{3}$, or $r \leq \frac{1}{12}$. This means that the contribution of agents 4 and 5 is at least $\frac{1}{4}+\frac{19}{60}-\frac{1}{12}=\frac{29}{60}$. But then agents $\{1,4,5\}$ would obviously be better off being together.

(i)-(iii) imply that a stable partition must consist of two jurisdictions. Thus, by (iv), there are only three (up to a permutation of agents in same locations) candidates to form a core stable partition: $P_{1}=\{1,2,3\} \bigcup\{4,5\}, P_{2}=\{1,2,4\} \bigcup\{3,5\}$, and $P_{3}=\{1,4,5\} \bigcup\{2,3\}$.

Consider $P_{1}$. Note that a group $\{1,2,4,5\}$ could make the first two members better off (relatively to $P_{1}$ ) by choosing $l$ to the left of $\frac{1}{12}$. In order for 4 and 5 to be better off, the following inequality should be satisfied: $\frac{1}{4}+\frac{19}{60}-l<\frac{1}{2}$ or $l>\frac{1}{15}$. Thus, agents $1,2,4,5$ indeed would be better off in $P_{1}$ by jointly choosing location $l$ within the interval $\left(\frac{1}{15}, \frac{1}{12}\right)$.

To complete our examination, note that agent 3 is better off joining 1,2, 4, rather than staying with 5 , whereas agent 1 is better off joining 2,3 , rather than staying with 4,5 . Thus, neither $P_{2}$ nor $P_{3}$ is stable.

Proof of Proposition 4.5: Define a function $\pi$ over the set $\mathcal{P}$ of all partitions of $N$ in such a way that for any partition $P=\left\{S_{k}\right\}_{1 \leq k \leq K} \in \mathcal{P}$, the value $\pi(P)$ is given by

$$
\pi(P)=\sum_{k=1}^{K}\left(D\left(S_{k}\right)+\sum_{r=1}^{\left|S_{k}\right|} \frac{1}{r}\right)
$$

Suppose that the function $\pi(\cdot)$ attains its minimum over $\mathcal{P}$ at $P=\left\{S_{k}\right\}_{1<k<K}$. (Due to the finiteness of $N$, this minimum always exists.) We will prove that $P$ is Nash stable, regardless of the choice of project locations $m_{k} \in M\left(S_{k}\right)$.

Assume that there is an agent $i \in S_{k} \in P$ and a jurisdiction $S_{t} \in P, t \neq k$, such that $i$ would be better off by joining the jurisdiction $S_{t}$, i.e.,

$$
c_{i}\left(m_{k}, S_{k}\right)>c_{i}\left(m^{\prime}, S_{t} \bigcup\{i\}\right),
$$

where $m^{\prime}=M\left(S_{t}\right) \cap M\left(S_{t} \bigcup\{i\}\right)$ is, by Remark 4.3, uniquely defined and is chosen according to $C M$ rule.

Denote by $P^{\prime} \in \mathcal{P}$ a partition that is obtained from $P$ by replacing $S_{k}$ and $S_{t}$ by $S_{k} \backslash\{i\}$ and $S_{t} \bigcup\{i\}$, respectively. We have

$$
\pi(P)-\pi\left(P^{\prime}\right)=\left(D\left(S_{k}\right)+\sum_{r=1}^{\left|S_{k}\right|} \frac{1}{r}+D\left(S_{t}\right)+\sum_{r=1}^{\left|S_{t}\right|} \frac{1}{r}\right)-
$$




$$
\begin{aligned}
& -\left(D\left(S_{k} \backslash\{i\}\right)+\sum_{r=1}^{\left|S_{k}\right|-1} \frac{1}{r}+D\left(S_{t} \bigcup\{i\}\right)+\sum_{r=1}^{\left|S_{t}\right|+1} \frac{1}{r}\right) \\
& =\left(D\left(S_{k}\right)-D\left(S_{k} \backslash\{i\}\right)\right)+\left(D\left(S_{t}\right)-D\left(S_{t} \bigcup\{i\}\right)\right)+\frac{1}{\left|S_{k}\right|}-\frac{1}{\left|S_{t}\right|+1} .
\end{aligned}
$$

Since $D\left(S_{k} \backslash\{i\}\right)$ is the minimum of the aggregate transportation costs in jurisdiction $S_{k} \backslash\{i\}$, we have

$$
\begin{aligned}
D\left(S_{k}\right) & =\sum_{j \in S_{k}}\left|m_{k}-l^{j}\right|=\left|m_{k}-l^{i}\right|+\sum_{j \in S_{k} \backslash\{i\}}\left|m_{k}-l^{j}\right| \\
& \geq\left|m_{k}-l^{i}\right|+\sum_{j \in S_{k} \backslash\{i\}}\left|m^{\prime \prime}-l^{j}\right|=\left|m_{k}-l^{i}\right|+D\left(S_{k} \backslash\{i\}\right),
\end{aligned}
$$

where $m^{\prime \prime} \in M\left(S_{k} \backslash\{i\}\right.$ ). (A specific choice of $m^{\prime \prime}$ does not matter here. For consistency reasons, we may assume that it also satisfies the $C M$ rule.) Moreover,

$$
D\left(S_{t} \bigcup\{i\}\right)=\sum_{j \in S_{t} \bigcup\{i\}}\left|m^{\prime}-l^{j}\right|=\left|m^{\prime}-l^{i}\right|+\sum_{j \in S_{t}}\left|m^{\prime}-l^{j}\right|=\left|m^{\prime}-l^{i}\right|+D\left(S_{t}\right) .
$$

Hence, we obtain

$$
\begin{gathered}
\pi(P)-\pi\left(P^{\prime}\right) \geq\left|m_{k}-l^{i}\right|-\left|m^{\prime}-l^{i}\right|+\frac{1}{\left|S_{k}\right|}-\frac{1}{\left|S_{t}\right|+1} \\
=\left(\left|m_{k}-l^{i}\right|+\frac{1}{\left|S_{k}\right|}\right)-\left(\left|m^{\prime}-l^{i}\right|+\frac{1}{\left|S_{t}\right|+1}\right)=c_{i}\left(m_{k}, S_{k}\right)-c_{i}\left(m^{\prime}, S_{t} \bigcup\{i\}\right)>0,
\end{gathered}
$$

which contradicts the initial assumption that the minimum of the function $\pi(\cdot)$ is attained at $P$.

Similarly, assume that there exists an agent $i \in S_{k}$ who is better off by forming a oneagent jurisdiction. This move would create a new partition $P^{\prime \prime}$ and we have

$$
\pi(P)-\pi\left(P^{\prime \prime}\right)=\left(D\left(S_{k}\right)+\sum_{r=1}^{\left|S_{k}\right|} \frac{1}{r}\right)-\left(D\left(S_{k} \backslash\{i\}\right)+\sum_{r=1}^{\left|S_{k}\right|-1} \frac{1}{r}+1\right) \geq
$$




$$
\begin{array}{r}
\left|m_{k}-l^{i}\right|+D\left(S_{k} \backslash\{i\}\right)+\sum_{r=1}^{\left|S_{k}\right|} \frac{1}{r}-\left(D\left(S_{k} \backslash\{i\}\right)+\sum_{r=1}^{\left|S_{k}\right|-1} \frac{1}{r}+1\right)= \\
\left(\left|m_{k}-l^{i}\right|+\frac{1}{\left|S_{k}\right|}\right)-1=c_{i}\left(m_{k}, S_{k}\right)-c_{i}(\{i\},\{i\})>0,
\end{array}
$$

again, leading to a contradiction.

\section{References}

[1] Alesina, A. and E. Spolaore (1997), On the number and size of nations, Quarterly Journal of Economics 112, 1027-1056.

[2] Aumann, R.J. and J.H. Drèze (1974), Cooperative games with coalition structure, International Journal of Game Theory 3, 217-237.

[3] Banerjee, S., Konishi, H., and T. Sönmez (2001), Core in a simple coalition formation game, Social Choice and Welfare 18, 135-153.

[4] Bogomolnaia, A. and M.O. Jackson (2002), The stability of hedonic coalition structures, Games and Economic Behavior 18, 201-230.

[5] Brams, S.J., Jones, M.A. and D.M. Kilgour (2002), Single-peakedness and disconnected coalitions, FEEM Working Paper \#19.2002.

[6] Cassela, A. (2001), The role of market size in the formation of jurisdictions, Review of Economic Studies 68, 83-108.

[7] Greenberg, J. and S. Weber (1986), Strong Tiebout equilibrium under restricted preferences domain, Journal of Economic Theory 38, 101-117.

[8] Greenberg, J. and S. Weber (1985), Multiparty equilibrium under proportional representation, American Political Science Review 79, 693-703.

[9] Jehiel, P. and S. Scotchmer (1997), Free mobility and the optimal number of jurisdictions, Annales d'Économie et de Statistique 45, 219-231.

[10] Jehiel, P. and S. Scotchmer (2001), Constitutional rules of exclusion in jurisdiction formation, Review of Economic Studies 68, 393-413.

[11] Haimanko, O., Le Breton, M. and S. Weber (2004a), Voluntary formation of communities for provision of public projects, Journal of Economic Theory 115, 1-34. 
[12] Haimanko, O., Le Breton, M. and S. Weber (2004b), Transfers in a polarized country: bridging the gap between efficiency and stability, Journal of Public Economics, forthcoming.

[13] Konishi, H., Le Breton, M., and S. Weber (1997), Pure strategy Nash equilibrium in a group formation game with positive externalities, Games and Economic Behavior 21, 161-182.

[14] Konishi, H., Le Breton, M., and S. Weber (1998), Equilibrium in a finite local public good economy, Journal of Economic Theory 79, 224-244.

[15] Le Breton, M. and S. Weber (2003), The art of making everybody happy: how to prevent a secession, IMF Stuff Papers, 50, 403-435.

[16] Le Breton, M. and S. Weber (2004), Secession-proof cost allocations and stable group structures in models of horizontal differentiation, in Group Formation in Economics: Networks, Clubs and Coalitions, Demange, G. and M. Wooders, eds., Cambridge University Press, 266-285.

[17] Le Breton, M., Weber, S. and J.H. Drèze (2004), The Rawlsian principle and secessionproofness in large heterogeneous societies, CORE Discussion Paper.

[18] Mas-Collel, A. (1980), Efficiency and decentralization in the pure theory of public goods, Quarterly Journal of Economics 94, 625-641.

[19] Monderer, D. and L.S. Shapley (1996), Potential games, Games and Economic Behavior 14, 124-143.

[20] Rosenthal, R. W. (1973), A class of games possessing pure-strategy Nash equilibrium, International Journal of Game Theory 2, 65-67. 